\title{
Psychosis in Azheimer's Disease
}

\author{
George M. Strickland M.D. \\ Tripler Army Medical Center, Honolulu, HI
}

Thomas F. Ditzler Ph.D.

Department of Psychiatry, Tripler Army Medical Center, Honolulu, Hl; Mental Health Programs, Center of Excellence in Disaster Management and Humanitarian Assistance, Honolulu, HI

Follow this and additional works at: https://jdc.jefferson.edu/jeffjpsychiatry

Part of the Psychiatry Commons

Let us know how access to this document benefits you

\section{Recommended Citation}

Strickland, George M. M.D. and Ditzler, Thomas F. Ph.D. (2002) "Psychosis in Azheimer's Disease," Jefferson Journal of Psychiatry. Vol. 17 : Iss. 1 , Article 4.

DOI: https://doi.org/10.29046/JJP.017.1.003

Available at: https://jdc.jefferson.edu/jeffjpsychiatry/vol17/iss1/4

This Article is brought to you for free and open access by the Jefferson Digital Commons. The Jefferson Digital Commons is a service of Thomas Jefferson University's Center for Teaching and Learning (CTL). The Commons is a showcase for Jefferson books and journals, peer-reviewed scholarly publications, unique historical collections from the University archives, and teaching tools. The Jefferson Digital Commons allows researchers and interested readers anywhere in the world to learn about and keep up to date with Jefferson scholarship. This article has been accepted for inclusion in Jefferson Journal of Psychiatry by an authorized administrator of the Jefferson Digital Commons. For more information, please contact: JeffersonDigitalCommons@jefferson.edu. 


\title{
Psychosis in Alzheimer's Disease
}

\author{
George M. Strickland, M.D. ${ }^{1}$ and \\ Thomas F. Ditzler, Ph.D. ${ }^{2}$
}

\begin{abstract}
Much of the basic science literature on Alzheimer's Disease (AD) reflects ongoing research into pathophysiology and neuropathology. Yet, despite reports of the association between psychotic symptoms and Alzheimer's disease, relatively little is known about why such symptoms develop in certain patients and not in others. Neuroimaging and genetic studies may provide greater understanding of this association and allow clinicians and researchers to prevent, predict and treat the onset of psychotic symptoms in the future. This paper will review the current literature on the topic of psychosis in Alzheimer's disease and focus on current recommendations for interventions by clinicians and caregivers.
\end{abstract}

\section{METHODOLOGY}

A literature search on the Medline database was performed. English language articles published between 1985-1999 were considered. Keywords "Alzheimer's Disease", "dementia", "psychosis", and "anti-psychotics" were used in different combinations. References in these articles led to further references utilized in this paper. Other sources include relevant textbook citations and recent journal articles from journals not available on the Medline database.

\section{INTRODUCTION}

Alzheimer's Disease (AD) represents the most common cause of dementia accounting for about $80 \%$ of all cases of dementia (1). Historically, AD was the first disease in which microscopic examination of the brain revealed the histopathologic changes of senile plaques, neurofibrillary tangles, and granulovacuolar degeneration of neurons (2). AD is a progressive, irreversible dementia that initially presents with memory problems followed by language, mathematical, visuospatial, and personality decline (1). The first symptom is usually amnesia or the inability to learn new information (1). Language difficulties begin with word-finding troubles followed by

\footnotetext{
${ }^{1}$ George M. Strickland, M.D. is a fourth year psychiatry resident at Tripler Army Medical Center, Honolulu, Hawaii.

${ }^{2}$ Thomas F. Ditzler, Ph.D. is Director of Research for the Department of Psychiatry, Tripler Army Medical Center, Honolulu, Hawaii and Liaison for Mental Health Programs, Center of Excellence in Disaster Management and Humanitarian Assistance, Honolulu, Hawaii.
} 
anomia and a fluent aphasia (1). This correlates to the neuropathology that is greatest in the regions posterior to Wernicke's area. Eventually the patient becomes mute and cannot comprehend (1). Visuospatial deficits and cognition changes occur which correlate to parietal and frontal injury (1). During the end-stages of the disease, the patient is unable to swallow or mobilize and death often occurs secondary to sepsis or dehydration. This article will focus on psychotic symptoms in $\mathrm{AD}$, but many other neuropsychiatric features like personality changes, depression, violence, sleep disturbance, and wandering are common (1).

$\mathrm{AD}$ causes profound emotional suffering and economic burden for patients and their caregivers (3). In 1997, the prevalence of AD in the US was 2.32 million with a range of 1.09 to 4.58 million (4). The lifetime risk of $\mathrm{AD}$ in the general population is roughly $15 \%$ (5). About $5 \%$ of individuals over 65 and $15-25 \%$ over 85 will develop AD (6). In the next 50 years the prevalence will almost quadruple, meaning that 1 in 45 Americans will have the disease (4). At present, the annual incidence is estimated to be 360,000 (4). In the US, treating AD costs 100 billion dollars a year, making it the third most expensive disease to treat after cancer and heart disease (7). The direct and total costs of $\mathrm{AD}$ to current and future generations are projected to reach 536 billion and 1.75 trillion dollars respectively (8).

\section{PSYCHOTIC SYMPTOMS}

Some studies have shown that approximately $30-50 \%$ of all Alzheimer's patients will eventually develop psychotic features $(9,10)$. Since a large number of AD patients will develop psychotic symptoms sometime over the course of their illness proper diagnosis and treatment is essential (11). Cooper, et al, noted that psychotic symptoms were more prevalent in advanced disease, but they can present at any time during the disease course (9). The link between psychotic symptoms and AD is well known. Dr. Alois Alzheimer's initial case report described symptoms of progressive memory loss, personality changes, delusional jealousy, paranoia, hallucinations, verbal outbursts, and disorientation in a 51 year-old woman $(2,12)$. Nonpsychotic disruptive behaviors such as motor restlessness, aggressive outbursts, pacing, and uncooperativeness are often mislabeled as psychotic or are assumed to be related to the underlying psychotic process (13). Thus, in any AD patient that suddenly develops hallucinations or delusions, other conditions need to be ruled out and include: drug intoxication, drug withdrawal, physical illness, depression, and delirium (3). Much of the management of Alzheimer's disease has focused on controlling these nonpsychotic disruptive behavioral symptoms, in part because they are the most distressing to caregivers and are the ones most likely to lead to institutionalization (14-16). Yet, this management often leads to trials of antipsychotics in patients without strictly defined psychotic processes (13).

\section{Delusions, Hallucinations, and Misidentifications}

The onset of psychosis in the $\mathrm{AD}$ patient can occur at any time, but is more likely in later stages of the disease $(11,17)$. The specific symptoms are wide ranging with 
the most common being delusions, hallucinations, and misidentifications $(18,19)$. Raskind noted that delusions in AD tend to be "unelaborated paranoid beliefs based on the underlying memory deficit", thereby differing from the "systematized complex delusions of primary thought disorders" (13). In a review of studies of psychotic symptoms among $\mathrm{AD}$ patients, delusions were identified in $10-73 \%$ of patients, with most studies showing a range of $30-38 \%$ (18). Delusions of the persecutory type were the most common, accounting for $30-33 \%$ of all delusions (18-20). A study of 170 patients by Deutsch, et al, found forty-three percent of patients had delusions with the following frequencies by type: persecutory, $73 \%$; reference, $14.9 \%$; jealousy, $9.5 \%$; grandiosity, $1.4 \%$; somatic, $1.4 \%$ (19).

The second most frequently occurring symptom of psychosis in $\mathrm{AD}$ is hallucinations. Multiple studies have found that visual hallucinations occur more often than auditory or tactile types $(18,19,21)$. Deutsch, et al, reported that $85 \%$ of all hallucinations were visual while Wragg and Jeste's review reports a median frequency of $13 \%$ for auditory hallucinations $(18,19)$. This pattern of greater visual hallucinations is consistent with conditions like delirium, drug intoxication, drug withdrawal, or psychosis secondary to a general medical condition. Schizophrenia, schizoaffective disorder, and mood disorders with psychotic features typically present with auditory hallucinations. These differences should provide the clinician with helpful clues in the diagnostic work-up of the psychotic patient.

A third type of psychotic symptom, misidentification syndromes or misperceptions, have been noted in many patients and are conceptualized as a form of delusion $(20,22,23)$. However, due their unique character, they have been categorized apart from delusions and hallucinations (24). Misperceptions result from a compromised capacity to organize perceptual information in the environment. Examples of misidentifications are the belief that a stranger is in the house, inability to recognize one's own reflection, or a belief that television shows are actually occurring in the house. Burns, et al, described a fourth form of misidentification, the erroneous identification of a friend or relative for another person (23). Misidentifications have been reported to occur in about one-quarter to one-third of $\mathrm{AD}$ patients with a median of $23 \%(20,23,25)$.

\section{Associated Psychiatric Symptoms}

When psychosis is seen with other neuropsychiatric symptoms like depression and agitation, the patient is more likely to be in the advanced stages of the disease and more difficult for the caregivers to manage (26). Levy, et al, longitudinally examined recurrence rates of neuropsychiatric symptoms in 181 patients with AD over a 1 -year period (17). Recurrence rates were $95 \%$ for psychosis, $93 \%$ for agitation, and $85 \%$ for depression. Of interest, the patients that displayed multiple symptoms at any point during the study showed greater recurrence rates of symptoms during the remaining part of the study. Also, if a patient displayed psychosis upon initial evaluation, they were more likely to display agitation and an accelerated cognitive decline over the next year. The patients in the 76-87 year group were noted to have more psychotic symptoms but less depression and agitation than younger patients. 
Women were found to display more neuropsychiatric symptoms than the men. This study concluded that once symptoms like psychosis, depression, and agitation begin that they frequently recur and that variances exist by sex and age (17).

Much of the literature reports that psychotic symptoms are associated with increased cognitive decline (27-32). Understanding the progression of cognitive decline in Alzheimer's disease and its association with psychosis is important because psychotic symptoms are one of the primary reasons for institutionalization $(15,21)$. In support of this, a study of 236 patients by Stern, et al, found that patients who demonstrated psychotic symptoms at the initial visit, increased their relative risk of reaching an institutional care rating equivalent to a nursing home by a factor of 1.5 (33). Lerner, et al, found that Alzheimer's patients with visual hallucinations performed worse on Mini-Mental Status Examinations and displayed more verbal outbursts, delusions, and paranoid ideation than those without hallucinations (29). In contrast to these findings, Wragg and Jeste, in their review of psychosis in $\mathrm{AD}$, cite several studies that found delusions were more likely in patients with higher cognitive scores (18). The explanation given is that some level of cognition is necessary for delusions to occur $(18,32)$. Teri, et al, found no difference in hallucination frequency at differing levels of cognitive functioning (34).

The relationship between particular psychotic symptoms and associated behaviors in Alzheimer's patients has also been examined $(9,19,29,35)$. Cooper, et al, reported that $\mathrm{AD}$ patients with psychosis were twice as likely to be agitated (9). Lerner, et al, found that visual hallucinations were positively associated with agitation and paranoid symptoms (29). Other behavioral signs and symptoms associated with visual hallucinations included auditory hallucinations, verbal outbursts, delusions, and paranoid ideation (29). In a report by Gilley, et al, hallucinations were associated with irritability, disinhibition, extrapyramidal symptoms, agitation, and delusions (35). Further, Deutch, et al, concluded that the presence of delusions and misidentifications might be predictive of physical aggression (19). Clearly, psychotic symptoms are associated with a wide range of behavioral reactions in Alzheimer's patients.

\section{Psychosis and Genetics}

Recently there has been a focus on the genetics of AD, which is a genetically heterogeneous disorder associated with 3 determinative genes and 1 susceptibility risk gene (5). The determinative genes are of the autosomal dominant type found on chromosomes 1, 14, and 21 are early-onset forms that manifest in the 40s and 50s, and account for about $5 \%$ of all cases (5). In 1993, an apolipoprotein E (APOE) epsilon 4 allele on chromosome 19 was discovered to have susceptibility to late-onset $\mathrm{AD}$ by increasing risk and decreasing age of onset $(5,36)$. The amyloid precursor protein (APP) gene is found on chromosome 21 is broken down to a protein, Beta-amyloid, that is the major constituent of senile plaques (6). Thus, persons with Down's Syndrome over 40 almost universally have pathological features of AD at autopsy (5). About $40 \%$ of patients with $\mathrm{AD}$ have a positive family history for the disorder, but the $50 \%$ concordance rate in monozygotic twins studies points to 
environmental factors $(6,36)$. Environmental risk studies, however, have given varied results when examining the areas of head trauma, sex, and toxins $(1,5)$.

Recent research has focused on the relationship between the APOE genotype and psychiatric symptoms to include psychosis (37-41). A study by Harwood, et al, that examined 501 patients with probable or possible AD found an elevated risk for psychosis among those with the epsilon 4 allele (37). Several other studies have found no correlation between the epsilon 4 allele and psychosis (38-40). Sweet, et al, examined the dopamine receptor gene polymorphisms DRD1, DRD2, DRD3, and DRD4 for associations with the presence of psychosis in AD (42). They found more psychosis in white patients with DRD1 B2/B2 homozygosity (42). Also, psychosis was more frequent in all AD patients with DRD3 1/1 or 2/2 homozygosity (42). This study concluded that genetic variation in DRD1 and DRD3 genes might modify the course of $\mathrm{AD}$, predisposing to the development of psychotic or aggressive symptoms (42).

\section{NEUROIMAGING}

Neuroimaging via computed tomography (CT) or magnetic resonance imaging (MRI) is not diagnostic for $\mathrm{AD}$, as $\mathrm{AD}$ produces no pathognomonic changes that are seen on conventional neuroimaging $(1,43)$. However, many experts recommend baseline imaging to rule out treatable forms of dementia. When used, non-contrast computed tomography to find atrophy or diffuse white matter is usually sufficient (43). Neuroanatomical correlates of severity of illness and presentation of behavioral symptoms have long been known in AD. Zubenko, et al, showed that psychotic AD patients had increased densities of senile plaques in the prosubiculum of the hippocampus and increased densities of neurofibrillary tangles in the middle frontal cortex when compared to non-psychotic AD patients at autopsy (44). Their findings are interesting to note as chronic idiopathic psychosis of earlier life is accompanied by decreased blood flow and glucose metabolism in the frontal cortex along with cell loss in the hippocampus (44).

Positron emission tomography (PET) and single emission computed tomography (SPECT) allow for visualization of brain abnormalities via new approaches to conventional imaging $(1,44)$. In the future PET and SPECT may allow for early prediction of which patients will develop psychosis. In general, AD causes reduced perfusion or metabolism in the region of the temporo-parieto-occipital junction as visualized by SPECT or PET $(1,45)$. AD patients show reduced perfusion and metabolism in the cortical association areas that spares the basal ganglia, thalamus, cerebellum, and primary cortex (3). Organic psychosis and schizophrenia have been hypothesized to be the result of temporal, parietal, and frontal lobe dysfunction; however, the neuroanatomic substrate for hallucinations and delusions is unknown at this time (10). Yet, many studies have found that delusions correlate with temporal and frontal lobe dysfunction on PET and SPECT (46). Kotrla, et al, examined the hypothesis that psychosis in patients with $\mathrm{AD}$ was associated with cerebral dysfunction in both the frontal and the parietal lobes, since virtually all AD patients have temporal lobe pathology (10). They found that delusional patients had hypoperfusion of the left frontal lobe while hallucinating patients had hypoperfusion 
in the parietal lobes. This study concluded that psychotic patients with AD had cerebral blood flow deficits significantly different from nonpsychotic patients with AD (10). Thus, AD may provide a model for investigation of the manifestations of the cerebral dysfunction that produces psychosis (10).

\section{MANAGEMENT}

If there is minimal potential danger to the patient or others, reassurance and distraction is the treatment of choice for psychotic AD patients (47). If agitation or violent behavior is present and places the patient or caregivers at risk for harm, pharmacologic treatment along with one-on-one care is often required. In some cases restraints may be required but ongoing use must be justified. The number one goal is patient and staff safety and comfort. Agitation, which often accompanies psychosis, may be due to an undiagnosed condition such as pain, anxiety, hunger, constipation, or fear of abandonment. Treatment here should focus on reassurance, attending to unmet needs and correcting the underlying condition with careful medical evaluation $(47,48)$.

\section{Neuroleptics}

The primary management of psychosis in Alzheimer's disease has traditionally relied upon neuroleptic medications that have long been used to target delusions, hallucinations, and agitation (14,15). The use of neuroleptics in the elderly population is complex because adverse effects are common and sensitivity to drug-drug interactions is increased (49). In this population the adverse effects of greatest concern include orthostatic hypotention, delirium, extra-pyramidal symptoms (EPS), tardive dyskinesia, urinary retention, and glaucoma. Sunderland noted that elderly demented patients may be more sensitive to the adverse effects of neuroleptic drugs and may require lower doses than non-demented controls (11). Of particular note is that the risk for tardive dyskinesia (TD) is $50 \%$ in those 65 years or older even with short-term use (49). Traditionally, low doses of high-potency agents like haloperidol have been the initial drugs of choice (50,51). Haloperidol may cause parkinsonism, making monitoring for bradykinesia, rigidity, and sialorrhea necessary (52). A randomized, placebo-controlled dose-comparison trial of haloperidol for psychosis and disruptive behaviors was conducted recently by Devanand (53). This study indicated a favorable therapeutic profile for doses of 2-3 mg/day of haloperidol, but a subgroup of patients developed EPS (53). The use of low-potency neuroleptics should generally be avoided due to their anti-cholinergic effects (15). Also, patients with Alzheimer's disease are at high risk for falls or may have chronic obstructive lung disease, urinary retention, constipation, or other conditions in which lowpotency agents are contraindicated $(14,15)$.

The development of newer medications such as risperidone and olanzapine, have made the drug of initial choice for psychosis an area of debate (54). Medications such as risperidone have gained favor in the treatment of schizophrenia and other psychotic disorders largely because of safer side-effect profiles. Risperidone has 
received support from some geriatric clinicians who report effectiveness at very low doses $(0.5-2.0 \mathrm{mg} /$ day) $(47,55)$. Several studies have shown risperidone to be effective in reducing psychotic symptoms among demented patients $(25,49,56)$. Katz, et al, examined 625 institutionalized patients with dementia (73\% with AD) and significant psychosis or behavioral symptoms in order to evaluate the efficacy and safety of risperidone in this population (56). The patients were randomized in a double-blind, placebo-controlled fashion to placebo, low-dose $(0.5 \mathrm{mg} / \mathrm{day})$, mediumdose ( $1 \mathrm{mg} /$ day), or high-dose ( $2 \mathrm{mg} /$ day) risperidone for 12 weeks (56). The study concluded that risperidone significantly improved symptoms of psychosis in severe dementia with $1 \mathrm{mg} /$ day being an appropriate dose for most patients (56). With regards to safety, the frequency of EPS was not significantly greater than placebo for those receiving $1 \mathrm{mg}$ /day of risperidone (56). Clozapine may prove useful for those patients sensitive to EPS, but due to its high cost, weekly to biweekly blood monitoring, and risk for agranulocytosis, its use may be limited in this population (55). Tariot reviewed reports of clozapine use in psychotic demented patients and found a mixed picture in terms of efficacy and side effects with some reports showing a reduction in psychosis and agitation while others showing no benefit (25). The elderly are more susceptible to bruising, sedation, hypotention, confusion, and other anti-cholinergic effects of clozapine. Most of the published literature for the use of clozapine in this population is based on open trials, chart reviews, and case reports. Thus, there is a lack of controlled trials.

Olanzapine and quetiapine are newer drugs that show similar efficacy to conventional neuroleptics and do not require blood monitoring (47). Olanzapine and quetiapine are not significantly different from placebo in regards to EPS profiles and have lower risk of tardive dyskenesia. Olanzapine has been shown to be generally safe in schizophrenic populations but may be accompanied by mild sedation, some anti-cholinergic effects, dizziness and weight gain (57). Quetiapine has been shown to cause no changes in hematological or ECG profiles, required no blood monitoring, and displays low EPS profiles (58). However, quetiapine demonstrates substantial histaminic receptor blockade with resulting dizziness, postural hypotension, somnolence, and weight gain in some patients (59). An interim analysis at 12 weeks of an ongoing one year, open trial of quetiapine in 151 elderly patients with dementia (75 patients with $\mathrm{AD}$ ) and mixed behavioral problems, including psychosis, was recently performed (60). Patients received $25 \mathrm{mg}$ to $800 \mathrm{mg} /$ day of quetiapine (median dose $=100 \mathrm{mg} /$ day) with $6 \%$ of patients showing EPS. This study concluded that quetiapine was well tolerated and associated with improvement in elderly patients with psychotic disorders (60).

Implementation and dosing of anti-psychotics agents is complex. In general, non-pharmacologic alternatives should be attempted first due to large numbers of potential side-effects of anti-psychotics. Generally, side-effect profiles dictate the choice of agent and can be minimized by using the lowest effective dose (47). Dosing should generally be started at about $1 / 4$ to $1 / 2$ the usual adult dose and risks versus benefits must be reassessed on an ongoing basis. For example with haloperidol, a starting dose of $1 \mathrm{mg}$ /day with gradual, upward dose titration is recommended (53). Use of anticholinergic agents should be avoided. Some authors suggest it may be 
more beneficial to schedule medication in anticipation of behavior rather than reacting to symptoms with as needed medication orders (51). In other words, identify specific times of day or activities that precipitate certain unwanted behavior and medicate about one hour prior to this time (25). Most commonly, this would be at night to help foster sleep and treat any behavioral problems that often peak at that time (47).

\section{Alternative Agents}

A wide variety of other agents have been used with some success, including lithium, beta-blockers, selective serotonin reuptake inhibitors (SSRI's), trazodone, buspirone, benzodiazapines, and anti-convulsants (47,50,51). Generally, these agents have been used to treat agitation or aggression rather than used to treat psychosis per se. For behavioral control, the use of trazodone seems promising in several studies (11). Trazodone given at bedtime reduces nighttime agitation, while lower doses during the day help with daytime agitation (52). Preliminary data suggest SSRI's may help alleviate agitation (47). Anti-convulsants may be indicated for mildly agitated patients who do not respond to anti-psychotic medication (47). Although anti-convulsants have fewer side-effects, blood monitoring is required. Benzodiazepines have been shown to control behavioral problems better than placebo but not as well as anti-psychotics (47). Benzodiazepines are often given in conjunction with anti-psychotics but should be used with caution in Alzheimer's patients. Generally, the use of low-dose, short-acting agents with no active metabolites (i.e.-oxazepam or lorazepam) are the initial drugs of choice. Unfortunately, side effects such as ataxia, confusion, and sedation may place the patient at risk for falls. None of the alternative agents have been found to target psychosis but may be helpful in treating associated agitation in appropriate cases (49).

Cummings describes the three pharmacologic treatments in use at present in the treatment of $\mathrm{AD}$ : disease-modifying agents, agents to manage symptoms of cognitive deficits, and psychotropic agents for behavioral disturbances (52). Both the clinical literature and the public have embraced vitamin E, estrogen, cholinesterase inhibitors and gingko biloba as possible disease-modifying agents or treatments for cognitive deficits (52). The present strategy is to treat mild-to-moderately affected patients with vitamin $\mathrm{E}$ and a cholinesterase inhibitor (i.e.- donepezil). The use of cholinesterase inhibitors appears to slow the rate of cognitive decline in some patients and may help to reduce hallucinations and delusions (52). Principal limitations of these agents include their expense and inability to stop the course of the disease. In later stages of the disease, psychotropic agents are usually necessary and prolongation of the illness with vitamin $\mathrm{E}$ may no longer be a therapeutic goal (52). Post-menopausal women on estrogen develop AD less often, and estrogen given to women who already have $\mathrm{AD}$ show reduced cognitive effects (52). No studies to date show whether or not those given estrogen are less likely to develop psychotic symptoms. There is some evidence that individuals administered non-steroidal anti-inflammatory drugs (NSAIDS) demonstrate lower incidence of AD, but little 
prospective data is available (52). No evidence to date has suggested the use of NSAIDS prevents the development of psychosis in AD patients.

\section{Non-pharmacologic Approaches}

The efficacy of nonpharmacologic interventions in the treatment of psychosis remains to be established and in many instances will have only limited benefit $(53,61,62)$. Yet, some sources stress that behavioral therapies are important in the management of $\mathrm{AD}$ as they may reduce the occurrence of psychotic symptoms in patients and decrease stress in caregivers $(14,15)$. Typically, specific behavioral techniques are organized around three central themes: 1) modification of the physical environment to minimize extraneous stimuli and maximize patient dignity and safety; 2) training of caregivers to communicate effectively with $\mathrm{AD}$ patients; and 3) provision of emotional support, community resource information, and respite activities for caregivers. Modifying the environment may be quite simple (26). For example, it is often helpful to arrange the home in an orderly and systematic way with notes to remind the patient where things are (26). Good lighting, careful placement of mirrors, and absence of televisions or radios can help reduce delusions and misidentifications (20). Since vision and hearing impairment often contribute to problems for $\mathrm{AD}$ patients it is important to ensure the patient's glasses and hearing aids are in good order. Assisting non-professional caregivers is vital in the treatment of $\mathrm{AD}$, as non-professionals provide $80 \%$ of overall care (26). Teaching family members to communicate effectively with the $\mathrm{AD}$ patient can ameliorate some of the frustration patients may evoke. Referring family members to the local chapter of the National Alzheimer's Disease \& Related Disorders Association can improve their access to community resources and overall quality of care $(26,52)$.

If the patient reaches the point where institutionalization becomes necessary, special care units are often available $(20,63)$. These units provide a safe, nonthreatening environment in which irrelevant stimuli are reduced (20). In recent years such units have become increasingly available and may slow the decline of functional capacity. A recent study of 1228 residents in 48 facilities with special care units demonstrated no statistically significant differences in speed of decline from traditional units; however, the authors concluded that these units might provide unmeasured benefits to families and residents by improving overall quality of life (63).

\section{CONCLUSIONS}

The association between psychotic symptoms and Alzheimer's disease is well known, and these symptoms are distressing to patients, caregivers, and clinicians. It is also known that delusions, hallucinations, and misidentifications occur commonly among $\mathrm{AD}$ patients and are associated with significant morbidity. Controversy remains as to whether or not psychotic symptoms lead to a more rapid cognitive decline, but most researchers agree that psychosis is positively associated with agitation and aggression $(9,19,29,35)$. 
Current management of psychosis centers on use of anti-psychotic medication, behavioral therapy, and family education $(14,15,20,50,51)$. There is a paucity of scientific literature on the nonpharmacologic approaches to the management of psychosis in $\mathrm{AD}$ and their overall efficacy is yet to be proven $(53,62)$. With regards to pharmacologic management, the use and development of newer medications with fewer side-effects appears promising (47). At the present time the literature lacks a number of controlled studies on the atypical anti-psychotics in AD patients, but it appears that more studies are forthcoming. The effects of special care units on functional outcome in $\mathrm{AD}$ is an area that requires more investigation (63). Current Research is now using functional neuroimaging to examine $\mathrm{AD}$, but neuroanatomic correlates of psychosis in $\mathrm{AD}$ remain largely unknown $(3,10)$. Articles addressing possible differences between psychotic symptoms in the familial forms and the susceptibility form of AD were not located in this review. The hope is that more will be discovered about possible genetic correlates to the neuropsychiatric symptoms of $\mathrm{AD}$ in the near future. It is apparent from this review that much work remains in the examination of the disease that Alzheimer described as, "a peculiar disease of the cerebral cortex" (2).

\section{REFERENCES}

1. Cummings JL, Coffey CE, (Eds) The American Psychiatric Press Textbook of Geriatric Neuropsychiatry. Washington DC; 1994; 11, 209, 390-404.

2. Spitzer RL, Gibbon M, Skodol AE, et al, (Eds) DSM-IV Casebook: A Learning Companion to the Diagnostic and Statistical Manual of Mental Disorders, Fourth Edition. Washington DC; 1994; 509-511.

3. Small GW. Differential diagnosis and early detection of dementia. Am J of Geriat Psychiatry 1998; 6(2), Suppl. 1: S26-S33.

4. Brookmeyer R, Gray S, Kawas C. Projections of Alzheimer's disease in the United States and public health impact of delaying disease onset. Am J of Public Health 1998; 88(9): 1337-1342.

5. Post SG, Whitehouse PJ, Binstock RH, et al. The clinical introduction of genetic testing for Alzheimer disease: an ethical perspective. JAMA 1997; 277: 832-836.

6. Kaplan HI, Sadock BJ. Synopsis of Psychiatry: Behavioral Sciences, Clinical Psychiatry, 8th ed. Williams and Wilkins; Baltimore; 1998; 328.

7. Snow C. Medicare HOMO's develop plan for future of Alzheimer's disease programming. Modern Healthcare 1996; 26(39): 67-70.

8. Ernst RL, Hay JW. The U.S. Economic and Social Costs of Alzheimer's Disease Revisited. Am J Public Health 1994; 84(8): 1261-1264.

9. Cooper JK, Mungras D, Verma M, et al. Psychotic symptoms in Alzheimer's disease. Int $\mathrm{J}$ of Geriat Psychiatry 1991; 6: 721-726.

10. Kotrla KJ, Chacko RC, Harper RG, et al. SPECT findings on psychosis in Alzheimer's disease. Am J of Psychiatry 1995; 152: 1470-1475.

11. Sunderland T. Treatment of the elderly suffering from psychosis and dementia. J of Clin Psychiatry 1996; 57, Suppl. 9: 53-56.

12. Alzheimer A. Uber eine Eigenartige Erkrankung der Hinrinde. Allegemeine Zeitschrift fur Psychiatrie und Psychisch. Gerichtlich Med 1907; 64:146-148; English translation in 
Bick K, Amaducci, L, Pepeu G, (Eds) The Early Story of Alzheimer's Disease. Padova, Liviana Press, 1987; 1-3.

13. Raskind MA. Treatment of Alzheimer's Disease and Other Dementias, in Schatzberg AF, Nemeroff CB, (Eds) The American Textbook of Psychopharmacology. Washington DC, $1995 ; 657-667$.

14. Loebel JP, Leibovici A. The management of other psychiatric states: hallucinations, delusions, and other disturbances. Med Clin of N Am 1994; 78(4): 841-859.

15. Wragg RE, Jeste DV. Neuroleptics and alternative treatments: management of behavioral symptoms and psychosis in Alzheimer's disease and related conditions. Psych Clinics of N Am 1988; 11(1): 195-213.

16. Devanand DP. Behavioral complications and their treatment in Alzheimer's disease. Geriatrics 1997; 52, Suppl. 2, September: S37-S39.

17. Levy ML, Cummings JL, Fairbanks LA, et al. Longitudinal assessment of symptoms of depression, agitation, and pyschosis in 181 patients with Alzheimer's disease. Am J of Psychiatry 1996; 153: 1438-1443.

18. Wragg RE, Jeste DV. Overview of depression and psychosis in Alzheimer's disease. Am J of Psychiatry 1989; 146: 577-587.

19. Duetsch LH, Bylsma FW, Rovner BW, et al. Psychosis and Physical Aggression in Probable Alzheimer's Disease. Am J of Psychiatry 1991; 148: 1159-1163.

20. Deutsch LH, Rovner BW. Agitation and other noncognitive abnormalities in Alzheimer's disease. Psychiatric Clin of N Am 1991; 14(2): 341-351.

21. Mendez MF, Martin RJ, Smyth KA, et al. Psychiatric symptoms associated with Alzheimer's disease. J of Neuropsychiatry and Clin Neurosciences 1990; 2: 28-33.

22. Rubin EH, Drevets WC, Burke WJ. The nature of psychotic symptoms in senile dementia of the Alzheimer type. J of Geriat Psychiatry and Neurology 1988; 1: 16-20.

23. Burns A, Jacoby R, Levy R. Psychiatric phenomena in Alzheimer's disease II: disorders of perception. Brit J of Psychiatry 1990; 157: 76-81.

24. Burns A. Psychiatric phenomena in dementia of the Alzheimer type. Inter Psychogeriatrics 1992; 4, Suppl. 1: 43-54.

25. Tariot PN. Treatment strategies for agitationa nd psychosis in dementia. J of Clin Psychiatry 1996; 57, Suppl. 14: 21-29.

26. Rabins PV, Mace NL, Lucas MJ. The impact of dementia on the family. JAMA 1982; 248: 333-335.

27. Drevets WC, Rubin EH. Psychotic symptoms and the longitudinal course of senile dementia of the Alzheimer type. Biol Psychiatry 1989; 25: 39-48.

28. Chui HC, Lyness SA, Sobel E, et al. Extrapyramidal signs and psychiatric symptoms predict faster cognitive decline in Alzheimer's disease. Archives of Neurology 1994; 51: $676-681$.

29. Lerner AJ, Koss E, Patterson MB, et al. Concomitants of visual hallucinations in Alzheimer's disease. Neurology 1994; 44: 523-527.

30. Rosen J, Zubenko GS. Emergence of psychosis and depression in the longitudinal evaluation of Alzheimer's disease. Biol Psychiatry 1991; 29: 224-232.

31. Mayeux R, Stern Y, Sano M. Psychosis in patients with dementia of the Alzheimer type. Ann of Neurology 1985; 18: 144.

32. Cummigns JL, Miller B, Hill MA, et al. Neuropsychiatric aspects of multi-infarct dementia and dementia of the Alzheimer type. Arch of Neurology 1987; 44: 389-393.

33. Stern Y, Tang MX, Albert MS, et al. Predicting time to nursing home care and death in individuals with Alzheimer disease. JAMA 1997; 277: 806-812. 
34. Teri L, Larson EB, Reifler BV. Behavioral disturbance in dementia of the Alzheimer's type. J of the Am Geriat Soc 1988; 36: 1-6.

35. Gilley DW, Whalen ME, Wilson RS, et al. Hallucinations and associated factors in Alzheimer's disease. J of Neuropsychiatry and Clin Neurosciences 1991; 3: 371-376.

36. Small GW. The pathogenesis of Alzheimer's disease. J of Clin Psychiatry 1998; 59, Suppl. 9: 7-14.

37. Harwood DG, Barker WW, Ownby RL, et al. Apolipoprotein-E (APO-E) genotype and symptoms of psychosis in Alzheimer's disease. Am J of Geriat Psychiatry 1999; 7(2): $119-123$.

38. Levy ML, Cummigns JL, Fairbanks LA, et al. Apolipoprotein E genotype and noncognitive symptoms in Alzheimer's disease. Biol Psychiatry 1999; 45(4): 422-5.

39. Lopez OL, Kamboh MI, Becker JT, et al. The apolipoprotein E episolon 4 allele is not associated with psychiatric symptoms of extrapyramidal signs in probable Alzheimer's disease. Neurology 1997; 49(3): 794-797.

40. Hirono N, Mori E, Yasuda M, et al. Factors associated with psychotic symptoms in Alzheimer's disease. J of Neurol, Neurosurg, and Psychiatry 1998; 64(5): 648-652.

41. Ramachandran G, Marder K, Tang M, et al. A preliminary study of apolipoprotein E genotype and psychiatric manifestatiosn of Alzheimer's disease. Neurology 1996; 47(1): $256-259$.

42. Sweet RA, Mimgaonkar VL, Kamboh MI, et al. Dopamine receptor genetic variation, psychosis, and aggression in Alzheimer disease. Arch of Neurol 1998; 55(10): 1335-1340.

43. Duncan BA, Siegal AP. Early diagnosis and management of Alzheimer's disease. J of Clin Psychiatry 1998; 59, Suppl. 9: 15-21.

44. Zubenko GS, Moossy J, Martinez AJ, et al. Neuropathologic and neurochemical correlates of psychosis in primary dementia. Arch of Neurol 1991; 48:619-624; correction, 1992; 49: 1064.

45. Holcomb HH, Links J, Smith C, et al. Positron emission tomography: measuring the metabolic and neurochemical characteristics of the living human nervous system, In: NC Andreasen (Ed) Brain Imaging: Applications: Applications in Psychiatry. Washington DC, American Psychiatric Press, 1989; pp. 235-370.

46. Cummings JL, Back C. The cholinergic hypothesis of neuropsychiatric symptoms in Alzheimer's disease. Am J of Geriat Psychiatry 1998; 6(2 suppl. 1): S64-S78.

47. American Psychiatric Association Practice Guidelines for The Treatment of Patients with Alzheimer's Disease and Other Dementias of Late Life. Am J of Psychiatry 1997; 154(5), Suppl: $1-39$.

48. Small GW, Rabins PV, Barry PP, et al. Diagnosis and treatment of Alzheimer disease and related disorders: consensus statement of the American Association for Geriatric Psychiatry, the Alzheimer's Association, and the American Geriatrics Society. JAMA 1997; 278: 1363-1371.

49. Zarate CA, Baldessarini RJ, Siegel AJ, et al. Risperidone in the elderly: a pharmacoepidemiologic study. J of Clin Psychiatry 1997; 58: 311-317.

50. Schneider LS, Pollock VE, Lyness SA. A metaanalysis of controlled trials of neuroleptic treatment in dementia. J of the Am Geriatr Soc 1990; 38: 553-563.

51. Hales RE, Yudofsky SC, Talbott JA, eds. American Psychiatric Press Textbook of Psychiatry, 2nd edition. Washington DC; 1994: 95-112.

52. Cummings JL. New developments in the treatment of Alzheimer's disease. Psychiatric Times 1998; 15(4): 50-54.

53. Devanand DP, Marder K, Michaels KS, et al. A randomized, placebo-controlled dose- 
comparison trial of haloperidol for psychosis and disruptive behaviors in Alzheimer's disease. Am J of Psychiatry 1998; 155(11): 1512-1520.

54. Masand PS, McEvoy JP. New antipsychotic agents should replace the old neuroleptic agents for the first-line management of psychosis. Journal of Psychotic Disorders: Reviews and Commentaries 1997; 1(4): 3,15.

55. Lacro JP, Jeste DV. Geriatric psychosis. Psychiatric Quarterly 1997; 68(3): 247-260.

56. Katz IR, Jeste DV, Mintzer JE, et al. Comparison of risperidone and placebo for psychosis and behavioral disturbances associated with dementia: a randomized, double-blind trial. Risperidone Study Group. J of Clin Psychiatry 1999; 60(2): 107-115.

57. Beasley CM, Tollefson GD, Tran PV. Safety of olanzapine. J of Clin Psychiatry 1997; 58, Suppl. 10: 13-17.

58. Arvanitis LA, Miller BG, Borison RL, et al. Multiple fixed doses of "seroquel" (quetiapine) in patients with acute exacerbation of schizophrenia: a comparison with haloperidol and placebo. Biol Psychiatry 1997; 42: 233-246.

59. Pies R. The horsemen are joined: the newer atypical antipsychotics. Psychiatric Times 1998; 15(1): 22-27.

60. McManus DQ, Arvanitis LA, Kowalcyk BB, for the Seroquel Trial Study Group. Quetiapine, a novel antipsychotic: experience in elderly patients with psychotic disorders. J of Clin Psychiatry 1999; 60: 292-298.

61. Leibovici A, Tariot PN. Agitation associated with dementia: a systemic approach to treatment. Psychopharmacology Bulletin 1988; 24: 49-53.

62. Schneider LS. Pharmacologic management of psychosis in dementia. J of Clin Psychiatry 1999; 60, Suppl. 8: 54-60.

63. Phillips CD, Sloane PD, Hawes C, et al. Effects of residence in Alzheimer disease special care units on functional outcomes. JAMA 1997; 278: 1340-1344. 